\title{
THE BINARY MATROIDS WITH NO 4-WHEEL MINOR
}

\author{
JAMES G. OXLEY
}

\begin{abstract}
The cycle matroids of wheels are the fundamental building blocks for the class of binary matroids. Brylawski has shown that a binary matroid has no minor isomorphic to the rank-3 wheel $M\left(\mathscr{W}_{3}\right)$ if and only if it is a series-parallel network. In this paper we characterize the binary matroids with no minor isomorphic to $M\left(\mathscr{W}_{4}\right)$. This characterization is used to solve the critical problem for this class of matroids and to extend results of Kung and Walton and Welsh for related classes of binary matroids.
\end{abstract}

1. Introduction. The purpose of this paper is to study the class of binary matroids with no minor isomorphic to $M\left(\mathscr{W}_{4}\right)$, the cycle matroid of the rank-4 wheel. The motivation for this study derives from the fact that for every 3-connected binary matroid $M$ with at least four elements, there is a sequence $M_{0}, M_{1}, M_{2}, \ldots, M_{n}$ of 3-connected matroids with $M_{n}=M$ such that each matroid in the sequence is a single-element deletion or contraction of its successor and, for some $r \geqslant 3, M_{0} \cong$ $M\left(\mathscr{W}_{r}\right)$. This result, a consequence of Tutte's wheels and whirls theorem [18], establishes the wheels as the fundamental nontrivial building blocks for the class of 3-connected binary matroids. Indeed, since every matroid that is not 3-connected is a direct sum or a 2-sum of two matroids on fewer elements (Theorem 1.2), these building blocks are fundamental to the whole class of binary matroids. It is natural then to consider which binary matroids can arise when, for some $r, M\left(\mathscr{W}_{r}\right)$ is excluded as a minor. For $r=3$, this question was answered by Brylawski [3] who identified the class of such matroids as the class of series-parallel networks. In this paper we characterize the corresponding class of matroids when $r=4$ by listing its 3-connected members. For larger values of $r$ it appears that a similar characterization will be much more difficult to obtain.

The matroid terminology used here will in general follow Welsh [21]. The ground set and rank of the matroid $M$ will be denoted by $E(M)$ and $\operatorname{rk} M$, respectively. If $T \subseteq E(M)$, rk $T$ will denote the rank of $T$. The deletion and contraction of $T$ from $M$ will be denoted by $M \backslash T$ and $M / T$, respectively. If $Z$ is an $n$-element circuit of $M$, then we shall call $Z$ an $n$-circuit; $Z$ is an odd circuit if $n$ is odd and an even circuit otherwise.

Received by the editors November 18,1985 .

1980 Mathematics Subject Classification (1985 Revision). Primary 05B35; Secondary 05C15, 05C35.

This research was partially supported by the National Science Foundation under Grant No. DMS8500494. 
A matroid $M$ is 3-connected if it is connected and $E(M)$ cannot be partitioned into subsets $X$ and $Y$ each having at least two elements such that $\operatorname{rk} X+\operatorname{rk} Y-$ $\operatorname{rk} M=1$. It is routine to verify that $M$ is 3-connected if and only if its dual $M^{*}$ is 3-connected.

If $M_{1}$ and $M_{2}$ are matroids on the sets $S$ and $S \cup e$ where $e \notin S$, then $M_{2}$ is an extension of $M_{1}$ if $M_{2} \backslash e=M_{1}$, and $M_{2}$ is a lift of $M_{1}$ if $M_{2}^{*}$ is an extension of $M_{1}^{*}$. We call $M_{2}$ a nontrivial extension of $M_{1}$ if $e$ is neither a loop nor a coloop of $M_{2}$ and $e$ is not in a 2-circuit of $M_{2}$. Likewise, $M_{2}$ is a nontrivial lift of $M_{1}$ if $M_{2}^{*}$ is a nontrivial extension of $M_{1}^{*}$. The following result is well known (see, for example, [12, Lemma 2.1]).

(1.1) LEMMA. Let $N$ be a 3-connected matroid having at least three elements and $M$ be an extension of $N$. Then $M$ is 3-connected if and only if $M$ is a nontrivial extension of $N$.

We shall assume familiarity with the operation of parallel connection of matroids; a detailed discussion of this operation and its properties can be found in [3]. For matroids $M_{1}$ and $M_{2}$ such that $E\left(M_{1}\right) \cap E\left(M_{2}\right)=\{p\}$, we shall denote the parallel connection of $M_{1}$ and $M_{2}$ with respect to the basepoint $p$ by $P\left(\left(M_{1}, p\right),\left(M_{2}, p\right)\right)$. The following basic link between 3-connection and parallel connection was proved by Seymour $[15,(2.6)]$.

(1.2) THEOREM. A connected matroid $M$ is not 3-connected if and only if there are matroids $M_{1}$ and $M_{2}$ each of which has at least three elements and is isomorphic to a minor of $M$ such that $M=P\left(\left(M_{1}, p\right),\left(M_{2}, p\right)\right) \backslash p$ where $p$ is not a loop or a coloop of $M_{1}$ or $M_{2}$.

When $M$ decomposes as in this theorem, it is called the 2-sum of $M_{1}$ and $M_{2}$.

If $\{x, y\}$ is a circuit of the matroid $M$, we say that $x$ and $y$ are in parallel in $M$. If, instead $\{x, y\}$ is a cocircuit of $M$, then $x$ and $y$ are in series in $M$. The matroid $M^{\prime}$ is a parallel extension of $M$ if $M=M^{\prime} \backslash T$ and every element of $T$ is in parallel with some element of $M^{\prime}$ not in $T$. Series extensions are defined analogously. A matroid in which each connected component is obtained from a single-element matroid by a sequence of operations each of which is either a series or parallel extension is called a series-parallel network. A detailed investigation of the properties of such matroids can be found in [3].

If $A$ is a matrix with entries in a field $F$, then the matroid on the set of columns of $A$ that is induced by linear independence over $F$ will be called the dependence matroid $D(A)$ of $A$. A basic tool in this paper is the well-known fact (see, for example, [5, Theorem 3.7]) that binary matroids are uniquely representable, that is, if $A$ and $A^{\prime}$ are $r \times n$ matrices over GF(2) such that the map which, for all $i$ in $\{1,2, \ldots, n\}$, takes the $i$ th column of $A$ to the $i$ th column of $A^{\prime}$ is an isomorphism from $D(A)$ to $D\left(A^{\prime}\right)$, then $A^{\prime}$ can be transformed into $A$ by a sequence of operations each of which consists of interchanging two rows or adding one row to another. 
Another result that we shall rely on heavily is the following easy consequence of Seymour's splitter theorem [15, (7.3)]. For $r \geqslant 2, \mathscr{W}^{r}$ will denote the whirl of rank $r$ $[21$, p. 81].

(1.3) THEOREM. Let $M$ and $N$ be 3-connected matroids such that $N$ is a minor of $M$, $|E(N)| \geqslant 4$, and if $N \cong M\left(\mathscr{W}_{k}\right), M$ has no $M\left(\mathscr{W}_{k+1}\right)$-minor, while if $N \cong \mathscr{W}^{k}, M$ has no $\mathscr{W}^{k+1}$-minor. Then there is a sequence $M_{0}, M_{1}, M_{2}, \ldots, M_{n}$ of 3-connected matroids such that $M_{0} \cong N, M_{n}=M$ and, for all $i$ in $\{1,2, \ldots, n\}, M_{i}$ is an extension or lift of $M_{i-1}$.

In $\$ 2$ of this paper, we state and prove the main theorem of the paper, a characterization of the binary 3-connected matroids with no $M\left(\mathscr{W}_{4}\right)$-minor. We also characterize a somewhat larger class of 3-connected binary matroids and, in doing so, use the following well-known result of Tutte [17]. The Fano matroid will be denoted $F_{7}$.

(1.4) THEOREM. A binary matroid is regular if and only if it has no minor isomorphic to $F_{7}$ or $F_{7}^{*}$.

In $\S 3$, we use the main theorem to determine a best-possible upper bound on the number of elements in a rank-r simple matroid with no $M\left(\mathscr{W}_{4}\right)$-minor. We then use this bound to extend a result of Kung [9]. In addition, we extend a result of Murty [11].

Let $M$ be a loopless matroid that is isomorphic to the dependence matroid $D(A)$ of some matrix $A$ over $\mathrm{GF}(q)$. If $A$ has $r$ rows, then $r \geqslant \operatorname{rk} M$ and the set $S$ of distinct columns of $A$ is a subset of $V(r, q)$. The critical exponent $c(M ; q)$ of $M$ is the least number $k$ of hyperplanes $H_{1}, H_{2}, \ldots, H_{k}$ of $V(r, q)$ such that $\left(\bigcap_{i=1}^{k} H_{i}\right) \cap S$ $=\varnothing$, this number being independent of the particular matrix $A$ representing $M$ [6]. The problem of determining $c(M ; q)$ is known as the critical problem [6, Chapter 16] for $M$. In the fourth and final section of this paper, we use our main theorem to solve the critical problem for the class of binary matroids with no $M\left(\mathscr{W}_{4}\right)$-minor. We also use results from $\S 3$ to solve the critical problem for a related class of binary matroids thereby extending two results of Walton and Welsh [20].

2. The main result. In this section we state and prove the main result of the paper, a characterization of all binary 3-connected matroids having no $M\left(\mathscr{W}_{4}\right)$-minor. We shall denote by $\Theta$ the class of all such matroids. It follows from Theorem 1.2 that one can construct every binary matroid with no $M\left(\mathscr{W}_{4}\right)$-minor by beginning with the members of $\Theta$ and repeatedly using the operations of 2-sum and direct sum.

Let $r$ be an integer exceeding two and $A_{r}$ be the following $r \times(2 r+1)$ matrix over GF(2):

$$
A_{r}=\left[\begin{array}{cccccccccccc}
a_{1} & a_{2} & \cdots & a_{r} & & b_{1} & b_{2} & b_{3} & \cdots & b_{r} & c_{r} \\
& & & & 0 & 1 & 1 & \cdots & 1 & 1 \\
& & & & 1 & 0 & 1 & \cdots & 1 & 1 \\
& & I_{r} & & 1 & 1 & 0 & \cdots & 1 & 1 \\
& & & & \vdots & \vdots & \vdots & & \vdots & \vdots \\
& & & & 1 & 1 & 1 & \cdots & 0 & 1
\end{array}\right]
$$


Let $Z_{r}$ be the dependence matroid of $A_{r}$. Then $Z_{3} \cong F_{7}$, the Fano matroid. Moreover, $Z_{r}^{*} \cong Z_{r+1} \backslash b_{r+1}, c_{r+1}$ for all $r \geqslant 3$. Hence, in particular, $F_{7}^{*} \cong Z_{3}^{*} \cong$ $Z_{4} \backslash b_{4}, c_{4}$. We also note that $Z_{4} \backslash c_{4} \cong A G(3,2)$. The following is the main result of the paper.

(2.1) Theorem. Let $M$ be a binary matroid. Then $M$ is 3-connected and has no $M\left(\mathscr{W}_{4}\right)$-minor if and only if

(i) $M \cong Z_{r}, Z_{r}^{*}, Z_{r} \backslash c_{r}$ or $Z_{r} \backslash b_{r}$ for some $r \geqslant 4$; or

(ii) $M \cong F_{7}, F_{7}^{*}$ or $M\left(\mathscr{W}_{3}\right)$; or

(iii) $M \cong U_{0,1}, U_{1,1}, U_{1,2}, U_{1,3}$ or $U_{2,3}$.

The only pairs of matroids in the above list that have the same rank and corank are the self-dual matroids $Z_{r} \backslash c_{r}$ and $Z_{r} \backslash b_{r}$. For $r \geqslant 4$, these matroids are not isomorphic since the latter has a 3-circuit whereas the former does not. We conclude that all the matroids listed are nonisomorphic.

To prove Theorem 2.1, we shall first characterize a somewhat larger class $\Psi$ of 3-connected binary matroids, namely those such matroids having no $P_{9}$ - or $P_{9}^{*}$-minor. Here $P_{9}$ denotes the extension of $M\left(\mathscr{W}_{4}\right)$ for which a binary representation is shown in Figure 1. The same figure also shows a Euclidean representation for $P_{9}$. In addition to the planes shown, there is one further 4-point plane. It contains the four circled points.

(2.2) THEOREM. Let $M$ be a binary matroid. Then $M$ is 3-connected having no minor isomorphic to $P_{9}$ or $P_{9}^{*}$ if and only if

(i) $M$ is regular and 3-connected;

(ii) $M \cong Z_{r}, Z_{r}^{*}, Z_{r} \backslash c_{r}$ or $Z_{r} \backslash b_{r}$ for some $r \geqslant 4$; or

(iii) $M \cong F_{7}$ or $F_{7}^{*}$.

Proof. Evidently neither $F_{7}$ nor $F_{7}^{*}$ has a minor isomorphic to $P_{9}$ or $P_{9}^{*}$. The fact that no regular matroid has such a minor follows, using Theorem 1.4 and duality, from the observation that the contraction of $(1,0,0,1)^{T}$ from $P_{9}$ has an $F_{7}$-minor. We now show that none of the matroids listed in (ii) has a $P_{9^{-}}$or a $P_{9}^{*}$-minor. Since both $P_{9}$ and $P_{9}^{*}$ have $M\left(\mathscr{W}_{4}\right)$ as a minor, it suffices to show that none of the matroids in (ii) has an $M\left(\mathscr{W}_{4}\right)$-minor. We shall use the following:

(2.3) LEMMA. The automorphism group of $Z_{r}$ is transitive on $\left\{a_{1}, a_{2}\right.$, $\left.\ldots, a_{r}, b_{1}, b_{2}, \ldots, b_{r}\right\}$. Moreover, for $r \geqslant 4$, every automorphism of $Z_{r}$ fixes $c_{r}$.

Proof. Interchanging rows $i$ and $j$ of $A_{r}$ induces an automorphism of $Z_{r}$ that swaps $a_{i}$ with $a_{j}$ and $b_{i}$ with $b_{j}$. Moreover, the automorphism of $Z_{r}$ that is induced by replacing row $i$ of $A_{r}$ by the sum of rows $i, 1$ and $r$ for each $i$ in $\{2,3, \ldots, r-1\}$ swaps $a_{1}$ with $b_{r}$ and $a_{r}$ with $b_{1}$. We conclude that the automorphism group of $Z_{r}$ is transitive on $\left\{a_{1}, a_{2}, \ldots, a_{r}, b_{1}, b_{2}, \ldots, b_{r}\right\}$. The fact that, for $r \geqslant 4$, every automorphism fixes $c_{r}$ follows immediately from the observation that, for such $r, c_{r}$ is the unique element of $Z_{r}$ that is in $r$ 3-circuits.

(2.4) LeMmA. $Z_{r}$ has no $M\left(\mathscr{W}_{4}\right)$-minor. 


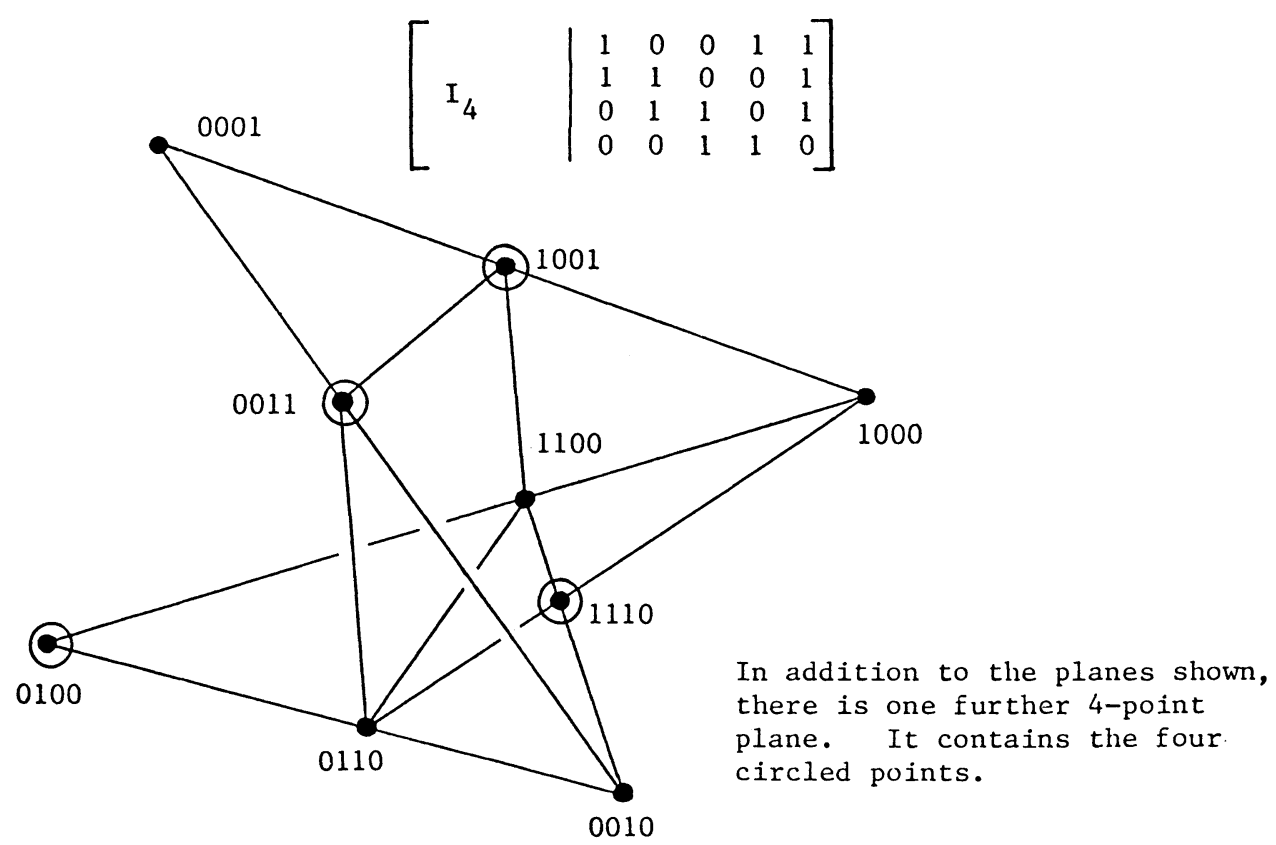

FIGURE 1

Proof. This is immediate for $r=3$. Now suppose that $r=4$. If $M\left(\mathscr{W}_{4}\right)$ is a minor of $Z_{4}$, it is a single-element deletion of $Z_{4}$. But, by the preceding lemma, $Z_{4}$ has precisely two nonisomorphic single-element deletions, namely $Z_{4} \backslash b_{4}$ and $Z_{4} \backslash c_{4}$. As $Z_{4} \backslash b_{4}, c_{4} \cong F_{7}^{*}$, neither $Z_{4} \backslash b_{4}$ nor $Z_{4} \backslash c_{4}$ is isomorphic to $M\left(\mathscr{W}_{4}\right)$. Thus the lemma holds for $r \leqslant 4$.

Now assume the lemma holds for $Z_{r}$ and consider $Z_{r+1}$ where $r \geqslant 4$. Suppose that $Z_{r+1}$ has an $M\left(\mathscr{W}_{4}\right)$-minor. Then, since rk $Z_{r+1}>\operatorname{rk} M\left(\mathscr{W}_{4}\right)$, the Scum Theorem [6] implies that for some element $z$ of $Z_{r+1}, Z_{r+1} / z$ has an $M\left(\mathscr{W}_{4}\right)$-minor. Now $Z_{r+1} / a_{r+1}$ is a parallel extension of $Z_{r}$ and therefore has no $M\left(\mathscr{W}_{4}\right)$-minor. Hence, $z \neq z_{r+1}$. Therefore, by the preceding lemma, $z \notin\left\{a_{1}, a_{2}, \ldots, a_{r+1}\right.$, $\left.b_{1}, b_{2}, \ldots, b_{r+1}\right\}$. Thus $z=c_{r+1}$. It is routine to check that $Z_{r+1} / c_{r+1} \cong M\left(C_{r+1}^{2}\right)$ where $C_{r+1}^{2}$ is the graph obtained by replacing every element of an $(r+1)$-circuit by two elements in parallel. Since $M\left(C_{r+1}^{2}\right)$ clearly has no $M\left(\mathscr{W}_{4}\right)$-minor, we have a contradiction. The lemma now follows by induction.

The last lemma completes the proof that none of the matroids listed in (2.2) (i)-(iii) has a $P_{9}$ - or a $P_{9}^{*}$-minor. We now prove that if $M$ has no such minor and is 3-connected and binary, then $M$ is listed in (i)-(iii). Suppose that $M$ is not regular. Then, by Theorem 1.4, $M$ has a minor isomorphic to $F_{7}$ or $F_{7}^{*}$. Thus, by Theorem 1.3 , there is a chain $M_{0}, M_{1}, \ldots, M_{n}$ of 3-connected matroids such that $M_{0} \cong F_{7}$ or $F_{7}^{*}, M_{n}=M$, and for all $i$ in $\{0,1,2, \ldots, n-1\}, M_{i}$ is a single-element deletion or contraction of $M_{i+1}$. For the rest of the proof of Theorem 2.2, we shall be concerned with the members of this chain. If $M \cong F_{7}$ or $F_{7}^{*}$, then $M$ is listed under (iii). Thus assume that this does not occur. We shall first suppose that $M_{0} \cong F_{7}^{*}$. Then, since $F_{7}^{*}$ has no nontrivial binary lifts, $M_{1}$ is a nontrivial extension of $F_{7}^{*}$. Seymour [16] 
has noted that $F_{7}^{*}$ has precisely two nonisomorphic binary nontrivial extensions, these two matroids being represented by the matrices $X_{4}$ and $Y_{4}$ shown below. Evidently these two matroids are

$$
X_{4}=\left[I_{4} \mid \begin{array}{llll}
0 & 1 & 1 & 1 \\
1 & 0 & 1 & 1 \\
1 & 1 & 0 & 1 \\
1 & 1 & 1 & 0
\end{array}\right], \quad Y_{4}=\left[I_{4} \mid \begin{array}{llll}
0 & 1 & 1 & 1 \\
1 & 0 & 1 & 1 \\
1 & 1 & 0 & 1 \\
1 & 1 & 1 & 1
\end{array}\right]
$$

isomorphic to $Z_{4} \backslash c_{4}$ and $Z_{4} \backslash b_{4}$. Thus, if $M_{0} \cong F_{7}^{*}$, then $M_{1} \cong Z_{4} \backslash c_{4}$ or $Z_{4} \backslash b_{4}$. But both of the last two matroids are isomorphic to their duals and therefore, if $M_{0} \cong F_{7}$, we again get that $M_{1} \cong Z_{4} \backslash c_{4}$ or $Z_{4} \backslash b_{4}$. We shall treat the cases when $M_{1} \cong Z_{4} \backslash b_{4}$ and when $M_{1} \cong Z_{4} \backslash c_{4}$ separately. First suppose that $M_{1} \cong Z_{4} \backslash c_{4}$.

(2.5) LEMma. Every nontrivial binary extension of $Z_{4} \backslash c_{4}$ is isomorphic to $Z_{4}$.

Proof. Let $N$ be a nontrivial binary extension of $Z_{4} \backslash c_{4}$. Then $N$ can be represented by the matrix obtained by adjoining the column $\left(x_{1}, x_{2}, x_{3}, x_{4}\right)^{T}$ to $X_{4}$ where each of $x_{1}, x_{2}, x_{3}$ and $x_{4}$ is in $\{0,1\}$. Evidently exactly two or exactly four of $x_{1}, x_{2}, x_{3}$ and $x_{4}$ are equal to 1 . Moreover, by the symmetry of $X_{4}$, all six matroids that arise by adjoining a column with exactly two ones are isomorphic. In addition, each of these matroids is isomorphic to the matroid $Z_{4}$ which is obtained when $\left(x_{1}, x_{2}, x_{3}, x_{4}\right)=(1,1,1,1)$. To see this, observe that by adding row 3 to each of rows 1 and 2 in the matrix $A_{4}$, we get the following matrix:

$$
\left[\begin{array}{lllllllll}
1 & 0 & 1 & 0 & 1 & 0 & 1 & 0 & 0 \\
0 & 1 & 1 & 0 & 0 & 1 & 1 & 0 & 0 \\
0 & 0 & 1 & 0 & 1 & 1 & 0 & 1 & 1 \\
0 & 0 & 0 & 1 & 1 & 1 & 1 & 0 & 1
\end{array}\right]
$$

On deleting the last column of this and suitably reordering the first eight columns, we get the matrix $X_{4}$.

We now suppose that $M_{1} \cong Z_{4} \backslash b_{4}$. The next lemma is stronger than we need for the proof of Theorem 2.2. We shall use this additional strength in the proof of Theorem 2.1.

(2.6) Lemma. The only column that can be adjoined to the matrix $Y_{4}$ to give a representation of a 3-connected binary matroid with no $M\left(\mathscr{W}_{4}\right)$-minor is $(1,1,1,0)^{T}$. Thus, the unique extension of $Z_{4} \backslash b_{4}$ in $\Theta$ is $Z_{4}$. Moreover, every other nontrivial binary extension of $Z_{4} \backslash b_{4}$ is isomorphic to $P_{9}$.

Proof. Assume that the column $\left(x_{1}, x_{2}, x_{3}, x_{4}\right)^{T}$ is adjoined to $Y_{4}$ to give a matrix $Y_{4}^{\prime}$ over $\mathrm{GF}(2)$ representing a 3-connected matroid $N$. If $\left(x_{1}, x_{2}, x_{3}, x_{4}\right)=(1,1,1,0)$, then clearly $N \cong Z_{4}$. We may now assume that $\left(x_{1}, x_{2}, x_{3}, x_{4}\right)$ has exactly two ones. Moreover, by the symmetry of $Y_{4}$, we can suppose that $\left(x_{1}, x_{2}, x_{3}, x_{4}\right)$ is one of $(1,1,0,0)$ and $(1,0,0,1)$. In each case, it is not difficult to check that the matrix obtained by deleting the sixth column from $Y_{4}^{\prime}$ represents a matroid isomorphic to $M\left(\mathscr{W}_{4}\right)$ and that $Y_{4}^{\prime}$ itself represents a matroid isomorphic to $P_{9}$. 
As an immediate consequence of the preceding lemma, we have the following corollary.

(2.7) COROllary. Every nontrivial binary extension of $Z_{4}$ has a $P_{9}$-minor and hence has an $M\left(\mathscr{W}_{4}\right)$-minor. Hence, for all $r \geqslant 4$, every nontrivial binary extension of $Z_{r}$ has a $P_{9}$-minor.

Now either (a) $M_{2}$ is an extension of $M_{1}$, or (b) $M_{2}$ is a lift of $M_{1}$. By Lemmas 2.5 and 2.6 and Corollary 2.7, in case (a), $M_{2} \cong Z_{4}$ and $M_{3}$ is a lift of $M_{2}$. By duality, in case (b), $M_{2} \cong Z_{4}^{*}$ and $M_{3}$ is an extension of $M_{2}$. In general, $Z_{r}^{*}$ is represented by the matrix

$$
A_{r}^{*}=\left[\begin{array}{ccccccccccc}
b_{1} & b_{2} & \cdots & b_{r} & c_{r} & & a_{1} & a_{2} & a_{3} & \cdots & a_{r} \\
& & & & & 0 & 1 & 1 & \cdots & 1 \\
& & & & 1 & 0 & 1 & \cdots & 1 \\
& & I_{r+1} & & & 1 & 1 & 0 & \cdots & 1 \\
& & & & & \vdots & \vdots & \vdots & & \vdots \\
& & & & & 1 & 1 & 1 & \cdots & 0 \\
& & & & & & 1 & 1 & 1 & \cdots & 1
\end{array}\right]
$$

The next lemma consider the nontrivial binary extensions of $Z_{r}^{*}$.

(2.8) Lemma. Suppose that $r \geqslant 4$ and that $x=\left(x_{1}, x_{2}, \ldots, x_{r+1}\right)^{T}$ is a column that is adjoined to $A_{r}^{*}$ to give a matrix $B$ over $\mathrm{GF}(2)$ representing a 3-connected matroid $N$. Then either (i) $N$ has a $P_{9}$-minor, or (ii) $x_{1}=x_{2}=\cdots=x_{r}=1$.

Proof. As $\left(x_{1}, x_{2}, \ldots, x_{r+1}\right)^{T}$ is distinct from every column of $A_{r}^{*}$, it has at least two ones. We may also assume it has at least two zeros otherwise (ii) holds. We now choose $i_{1}, i_{2}, i_{3}$ and $i_{4}$ to be distinct elements of $\{1,2, \ldots, r\}$ such that $x_{i_{1}}=1$, $x_{i_{2}}=0$ and $x_{i_{3}}=1+x_{r+1}$, the sum here being taken modulo 2 . Then exactly two of $x_{i_{1}}, x_{i_{2}}, x_{i_{3}}$ and $x_{r+1}$ are 1 . To construct a matrix representing a $P_{9}$-minor of $N$, we proceed as follows. First delete all the rows of $B$ except $i_{1}, i_{2}, i_{3}$ and $r+1$, and arrange the remaining rows in the order listed. Then delete all the columns of the resulting matrix except $b_{i_{1}}, b_{i_{2}}, b_{i_{3}}, c_{r}, a_{i_{1}}, a_{i_{2}}, a_{i_{3}}, a_{i_{4}}$ and $x$, and arrange the remaining columns in the order listed. The matrix obtained by this construction is $Y_{4}$ with one column adjoined, this extra column having two ones and two zeros. We conclude, using Lemma 2.6 , that $N$ has a $P_{9}$-minor.

On adjoining $(1,1,1, \ldots, 1,0)^{T}$ to $Z_{r}^{*}$, we obtain a matrix representing $Z_{r+1} \backslash c_{r+1}$; on adjoining $(1,1,1, \ldots, 1,1)^{T}$ instead, or adjoining both these columns, we get representations for $Z_{r+1} \backslash b_{r+1}$ and $Z_{r+1}$, respectively. It follows from this, using a straightforward induction argument and Lemma 1.1, that for every $r \geqslant 4$, all of $Z_{r}$, $Z_{r}^{*}, Z_{r} \backslash b_{r}$ and $Z_{r} \backslash c_{r}$ are 3-connected. The following result is an easy consequence of these observations and the last lemma.

(2.9) COROllary. For $r \geqslant 4, Z_{r}^{*}$ has precisely two nonisomorphic extensions in $\Psi$, these being isomorphic to $Z_{r+1} \backslash b_{r+1}$ and $Z_{r+1} \backslash c_{r+1}$. Moreover, the unique extension of each of the last two matroids that is in $\Psi$ is $Z_{r+1}$. 
The next result finishes the proof of Theorem 2.2.

(2.10) COROllary. Let $M_{0}, M_{1}, M_{2}, \ldots$ be a sequence of binary 3-connected matroids each of which is an extension or lift of its predecessor. Suppose also that $M_{0} \cong F_{7}$ or $F_{7}^{*}$ and that none of $M_{1}, M_{2}, \ldots$ has a $P_{9}$ or $P_{9}^{*}$-minor. Then, for all $j \geqslant 1, M_{2 j-1} \cong Z_{j+3} \backslash b_{j+3}$ or $Z_{j+3} \backslash c_{j+3}$ and $M_{2 j} \cong Z_{j+3}$ or $Z_{j+3}^{*}$.

Proof. We argue by induction on $j$. We noted earlier that $M_{1} \cong Z_{4} \backslash b_{4}$ or $Z_{4} \backslash c_{4}$ and that $M_{2} \cong Z_{4}$ or $Z_{4}^{*}$. Thus the result holds for $j=1$. Assume it holds for $j<k$ and let $j=k$. Then $M_{2 k-2} \cong Z_{k+2}$ or $Z_{k+2}^{*}$. In the latter case, by Corollaries 2.7 and 2.9, $M_{2 k-1}$ is an extension of $M_{2 k-2}$ and is isomorphic to $Z_{k+3} \backslash b_{k+3}$ or $Z_{k+3} \backslash c_{k+3}$. Since the last two matroids are isomorphic to their duals, if $M_{2 k-2} \cong Z_{k+2}$, then again $M_{2 k-1} \cong Z_{k+3} \backslash b_{k+3}$ or $Z_{k+3} \backslash c_{k+3}$. Now using Corollary 2.9 and duality, we get that $M_{2 k} \cong Z_{k+3}$ or $Z_{k+3}^{*}$ and the theorem follows by induction.

It is now straightforward to complete the proof of Theorem 2.1 and we omit the details.

3. A bound on the number of elements. Dirac [7] proved that, for all $n \geqslant 3$, a simple $n$-vertex graph with no subgraph homeomorphic from $\mathscr{W}_{3}$ has at most $2 n-3$ edges. As every binary matroid having no $M\left(\mathscr{W}_{3}\right)$-minor is a series-parallel network [3, Theorem 7.6] and hence is graphic, Dirac's result gives that, for all $r \geqslant 2$, a simple binary matroid of rank $r$ having no $M\left(\mathscr{W}_{3}\right)$-minor has at most $2 r-1$ elements. A similar linear bound on the number of elements in a rank-r simple ternary matroid having no $M\left(\mathscr{W}_{3}\right)$-minor was proved in [13, Theorem 5.1]. By making the obvious modifications to the proof of that result and using the main theorem of this paper,we get the next theorem. The details of the proof are omitted.

(3.1) THEOREM. Let $M$ be a simple binary matroid of rank $r$ having no $M\left(\mathscr{W}_{4}\right)$-minor. Then

$$
|E(M)| \leqslant \begin{cases}3 r-2, & \text { if } r \text { is odd }, \\ 3 r-3, & \text { if } r \text { is even. }\end{cases}
$$

Moreover, the following list includes all the matroids that attain this bound:

(i) $r=1, U_{1,1}$;

(ii) $r=2, U_{2,3}$;

(iii) $r=3, F_{7}$;

(iv) $r=4, Z_{4}, P\left(F_{7}, U_{2,3}\right)$;

(v) $r=2 t+1$ for $t \geqslant 2$, all matroids that can be formed from $t$ copies of $F_{7}$ using $t$ - 1 parallel connections;

(vi) $r=2 t$ for $t \geqslant 3$, all matroids that can be formed from $t-1$ copies of $F_{7}$ and one copy of $U_{2,3}$ using $t-1$ parallel connections, and all matroids that can be formed from $t-2$ copies of $F_{7}$ and one copy of $Z_{4}$ using $t-2$ parallel connections.

We now consider extending this result. We shall use some additional terminology. For a class $\mathscr{M}$ of matroids which contains a simple matroid of every nonzero rank, Kung [9] has defined the size function $h(\mathscr{M}, r)$ to be the function on $\mathbf{Z}^{+}$given by

$$
h(\mathscr{M}, r)=\max \{|E(M)|: M \in \mathscr{M}, \text { rk } M=r \text { and } M \text { is simple }\} .
$$


If $N_{1}, N_{2}, \ldots, N_{m}$ are matroids, then $\operatorname{EX}\left(N_{1}, N_{2}, \ldots, N_{m}\right)$ will denote the class of binary matroids having no minor isomorphic to any of $N_{1}, N_{2}, \ldots, N_{m}$. The following is an immediate consequence of the last theorem.

(3.2) Corollary.

$$
h\left(\operatorname{EX}\left(M\left(\mathscr{W}_{4}\right)\right), r\right)= \begin{cases}3 r-2, & \text { if } r \text { is odd }, \\ 3 r-3, & \text { if } r \text { is even } .\end{cases}
$$

The next result extends this corollary.

(3.3) THEOREM.

$$
h\left(\mathrm{EX}\left(M\left(K_{5}\right), P_{9}, P_{9}^{*}\right), r\right)= \begin{cases}3 r-2, & \text { if } r \text { is odd } . \\ 3 r-3, & \text { if } r \text { is even } .\end{cases}
$$

To prove this theorem we shall use both Corollary 3.2 and the next theorem. The latter is one of the several results of Kung $[9,10]$ on the size functions of various classes of binary matroids.

(3.4) THEOREM [9, THEOREM 9.1].

$$
h\left(\operatorname{EX}\left(M\left(K_{5}\right), F_{7}^{*}\right), r\right)= \begin{cases}3 r-2, & \text { if } r \text { is odd } . \\ 3 r-3, & \text { if } r \text { is even. }\end{cases}
$$

Proof OF Theorem 3.3. By [9, Lemma 10.1], since $\operatorname{EX}\left(M\left(\mathscr{W}_{4}\right)\right)$ and $\operatorname{EX}\left(M\left(K_{5}\right), F_{7}{ }^{*}\right)$ have the same size function, $\operatorname{EX}\left(M\left(\mathscr{W}_{4}\right)\right) \cup \operatorname{EX}\left(M\left(K_{5}\right), F_{7}{ }^{*}\right)$ also has this size function. To establish the theorem, we shall first show that

$$
\operatorname{EX}\left(M\left(\mathscr{W}_{4}\right)\right) \cup \operatorname{EX}\left(M\left(K_{5}\right), F_{7}^{*}\right) \subseteq \operatorname{EX}\left(M\left(K_{5}\right), P_{9}, P_{9}^{*}\right) .
$$

Since the class $\operatorname{EX}\left(M\left(\mathscr{W}_{4}\right)\right) \cup \operatorname{EX}\left(M\left(K_{5}\right), F_{7}^{*}\right)$ is closed under minors, it can certainly be characterized by excluded minors. It is straightforward to check that $M\left(K_{5}\right)$ is a minimal such excluded minor. The following lemma completes the proof of (3.5). We omit its routine proof.

(3.6) LEMMA. Both $P_{9}$ and $P_{9}^{*}$ are minor-minimal matroids not in $\operatorname{EX}\left(M\left(\mathscr{W}_{4}\right)\right) \cup$ $\operatorname{EX}\left(M\left(K_{5}\right), F_{7}^{*}\right)$.

The next lemma completes the proof of Theorem 3.3.

(3.7) Lemma. Let $M$ be a simple matroid that is in $\operatorname{EX}\left(M\left(K_{5}\right), P_{9}, P_{9}^{*}\right)$ but is not in $\operatorname{EX}\left(M\left(\mathscr{W}_{4}\right)\right) \cup \operatorname{EX}\left(M\left(K_{5}\right), F_{7}{ }^{*}\right)$. Then

$$
|E(M)| \leqslant \begin{cases}3 \mathrm{rk} M-2, & \text { if } \mathrm{rk} M \text { is odd }, \\ 3 \mathrm{rk} M-3, & \text { if } \mathrm{rk} M \text { is even. }\end{cases}
$$

Proof. Assume that the lemma is not true and let $N$ be a minor-minimal counterexample. It is straightforward to show, using Theorem 1.2, that $N$ must be 3-connected. Moreover, since $N \notin \operatorname{EX}\left(M\left(K_{5}\right), F_{7}^{*}\right)$ but $N \in \operatorname{EX}\left(M\left(K_{5}\right), P_{9}, P_{9}^{*}\right)$, $N$ has an $F_{7}{ }^{*}$-minor. Thus, by Theorem 1.3 , there is a chain $N_{0}, N_{1}, N_{2}, \ldots, N_{n}$ of 3-connected matroids such that $N_{0} \cong F_{7}{ }^{*}, N_{n}=N$ and, for each $i$ in $\{0,1,2, \ldots$, $n-1\}, N_{i}$ is a single-element deletion or contraction of $N_{i+1}$. By Theorem 3.1, $N=N_{n} \notin \operatorname{EX}\left(M\left(\mathscr{W}_{4}\right)\right)$. But $N_{0} \in \operatorname{EX}\left(M\left(\mathscr{W}_{4}\right)\right)$. Let

$$
m=\min \left\{i: N_{i} \notin \operatorname{EX}\left(M\left(\mathscr{W}_{4}\right)\right)\right\} .
$$


Then $N_{m-1} \in \operatorname{EX}\left(M\left(\mathscr{W}_{4}\right)\right)$ and, by Theorem 2.1, $N_{m-1}$ is isomorphic to $Z_{r}, Z_{r}^{*}$, $Z_{r} \backslash b_{r}$ or $Z_{r} \backslash c_{r}$ for some $r \geqslant 4$.

Now $N_{m}$ is an extension or lift of $N_{m-1}$. Since $Z_{r} \backslash b_{r}$ and $Z_{r} \backslash c_{r}$ are both isomorphic to their duals and $Z_{r}^{*} \cong Z_{r+1} \backslash b_{r+1}, c_{r+1}$, one of $N_{m}$ and $N_{m}^{*}$ is isomorphic to an extension of $N^{\prime}$ where $N^{\prime}$ is isomorphic to $Z_{r}, Z_{r} \backslash b_{r}$ or $Z_{r} \backslash c_{r}$ for some $r \geqslant 4$, or to $Z_{r} \backslash b_{r}, c_{r}$ for some $r \geqslant 5$. But, by Lemmas 2.5, 2.6 and 2.8 and Corollary 2.7, every nontrivial binary extension of each of the possibilities for $N^{\prime}$ has a $P_{9}$-minor or is in $\operatorname{EX}\left(M\left(\mathscr{W}_{4}\right)\right)$. Thus one of $N_{m}$ and $N_{m}^{*}$ has a $P_{9}$-minor. It follows that $N$ has a $P_{9}$ - or a $P_{9}^{*}$-minor; a contradiction.

To conclude this section, we determine the size function of $\operatorname{EX}\left(P_{9}, P_{9}^{*}\right)$ noting that, unlike $h\left(\operatorname{EX}\left(M\left(K_{5}\right), P_{9}, P_{9}^{*}\right), r\right)$, it is quadratic in $r$.

(3.8) THEOREM.

$$
h\left(\operatorname{EX}\left(P_{9}, P_{9}^{*}\right), r\right)= \begin{cases}\left(\begin{array}{c}
r+1 \\
2
\end{array}\right), & \text { if } r \neq 3, \\
\left(\begin{array}{c}
r+1 \\
2
\end{array}\right)+1, & \text { if } r=3 .\end{cases}
$$

This result extends the following theorem of Murty [11] (see also [1, 2]) which itself is an extension of Heller's result $[8]$ that $h\left(\operatorname{EX}\left(F_{7}, F_{7}^{*}\right), r\right)=\left(\begin{array}{c}r+1 \\ 2\end{array}\right)$.

(3.9) THEOREM. $h\left(\operatorname{EX}\left(F_{7}\right), r\right)=\left(\begin{array}{c}r+1 \\ 2\end{array}\right)$.

On combining Heller's result with Theorem 3.8, we get the size function for $\operatorname{EX}\left(F_{7}^{*}\right)$. This can also be deduced from a result of Walton [19].

(3.10) COROllary.

$$
h\left(\operatorname{EX}\left(F_{7}^{*}\right), r\right)= \begin{cases}\left(\begin{array}{c}
r+1 \\
2
\end{array}\right), & \text { if } r \neq 3, \\
\left(\begin{array}{c}
r+1 \\
2
\end{array}\right)+1, & \text { if } r=3 .\end{cases}
$$

Proof of Theorem 3.8. We argue by induction on $r$. As $P G(2,2) \cong F_{7} \in$ $\operatorname{EX}\left(P_{9}, P_{9}^{*}\right)$, it follows easily that $h\left(\operatorname{EX}\left(P_{9}, P_{9}^{*}\right), 3\right)=7=\left(\begin{array}{c}3+1 \\ 2\end{array}\right)+1$. To see that $h\left(\operatorname{EX}\left(P_{9}, P_{9}^{*}\right), r\right) \geqslant\left(\begin{array}{c}r+1 \\ 2\end{array}\right)$ for $r \neq 3$, we note that $M\left(K_{r+1}\right)$ is a rank-r member of $\mathrm{EX}\left(P_{9}, P_{9}^{*}\right)$. Evidently, for $r=1$ or $2, h\left(\mathrm{EX}\left(P_{9}, P_{9}^{*}\right), r\right)=\left(\begin{array}{c}r+1 \\ 2\end{array}\right)$. Hence, for $r \leqslant 3$, $h\left(\operatorname{EX}\left(P_{9}, P_{9}^{*}\right), r\right)$ is as stated in the theorem. Now suppose that $M$ is a simple rank-r member of $\operatorname{EX}\left(P_{9}, P_{9}^{*}\right)$ having $h\left(\operatorname{EX}\left(P_{9}, P_{9}^{*}\right), r\right)$ elements and assume that $r \geqslant 4$. If $M \in \operatorname{EX}\left(F_{7}\right)$, then, by Theorem 3.9, $h\left(\operatorname{EX}\left(P_{9}, P_{9}^{*}\right), r\right)=\left(\begin{array}{c}r+1 \\ 2\end{array}\right)$. Thus we can suppose that $M$ has an $F_{7}$-minor. Since $|E(M)|=h\left(\operatorname{EX}\left(P_{9}, P_{9}^{*}\right), r\right) \geqslant\left(\begin{array}{c}r+1 \\ 2\end{array}\right)$, it follows from Theorem 2.2 that $M$ is not 3-connected. Then using Theorem 1.2 and the choice of $M$, we can deduce that $M=P\left(N_{1}, N_{2}\right)$ where both $N_{1}$ and $N_{2}$ are simple members of $\operatorname{EX}\left(P_{9}, P_{9}^{*}\right)$ having rank at least two. It is now straightforward to apply the induction assumption to $N_{1}$ and $N_{2}$ to obtain the contradiction that $|E(M)| \leqslant$ $\left(\begin{array}{c}r+1 \\ 2\end{array}\right)-1$. 
4. The critical problem. A loopless binary matroid with no $M\left(\mathscr{W}_{3}\right)$-minor has critical exponent at most two [3]. In this section we shall show that a loopless binary matroid with no $M\left(\mathscr{W}_{4}\right)$-minor has critical exponent at most three and explicitly determine the critical exponents of all loopless binary 3-connected matroids with no $M\left(\mathscr{W}_{4}\right)$-minor. We shall use the following result.

(4.1) Lemma [4, THeOREM 10.3]. Let $M$ be a matroid that is represented by the matrix $\left[I_{r} \mid A\right]$ over $G F(2)$. Then the following statements are equivalent:

(i) $c(M ; 2)=1$.

(ii) $M$ has no odd circuits.

(iii) Every column of $A$ contains an odd number of nonzero entries.

(4.2) THEOREM. Let $M$ be a loopless binary 3-connected matroid having no $M\left(\mathscr{W}_{4}\right)$ minor. Then $c(M ; 2)=2$ unless

(i) $M$ has rank one, or $r$ is an even integer exceeding 3 and $M \cong Z_{r} \backslash c_{r}$ or $Z_{r-1}^{*}$; or

(ii) $r$ is an odd integer exceeding 2 and $M \cong Z_{r}$.

In cases (i) and (ii), $c(M ; 2)=1$ and 3 , respectively.

Proof. If $|E(M)| \leqslant 4$, then, by Theorem $2.1, M \cong U_{1,1}, U_{1,2}, U_{1,3}$ or $U_{2,3}$ and it is easy to check that $c(M ; 2)$ is as claimed. Now suppose that $|E(M)|>4$. Then, for some $r \geqslant 3, M$ is isomorphic to one of $Z_{r}, Z_{r}^{*}, Z_{r} \backslash b_{r}$ or $Z_{r} \backslash c_{r}$. We shall treat the cases when $r$ is even and when $r$ is odd separately. First suppose that $r$ is even. The matroid $Z_{r} \backslash c_{r}$ is represented by the matrix $A_{r}^{\prime}$ obtained from $A_{r}$ by deleting the last column. Every column of $A_{r}^{\prime}$ has an odd number of nonzero entries and so, by Lemma 4.1, $c\left(Z_{r} \backslash c_{r} ; 2\right)=1$. It follows that $c\left(Z_{r} ; 2\right) \leqslant 2$ and hence $c\left(Z_{r} \backslash b_{r} ; 2\right) \leqslant 2$. Moreover, since $Z_{r-1}^{*} \cong Z_{r} \backslash c_{r}, b_{r}, c\left(Z_{r-1}^{*} ; 2\right)=1$. Now consider $Z_{r} \backslash b_{r}$. It has an odd circuit, namely $\left\{a_{1}, a_{2}, \ldots, a_{r}, c_{r}\right\}$. Therefore, by Lemma 4.1, $c\left(Z_{r} \backslash b_{r} ; 2\right) \geqslant 2$ and hence $c\left(Z_{r} ; 2\right) \geqslant 2$. We conclude that

$$
c\left(Z_{r} \backslash b_{r} ; 2\right)=c\left(Z_{r} ; 2\right)=2 .
$$

Next we suppose that $r$ is odd. Then $Z_{r-1}^{*}$ has an odd circuit, namely $\left\{a_{1}, b_{2}, b_{3}, \ldots, b_{r-1}, c_{r-1}\right\}$. Therefore $c\left(Z_{r-1}^{*} ; 2\right) \geqslant 2$. Moreover, as both $Z_{r} \backslash b_{r}$ and $Z_{r} \backslash c_{r}$ are extensions of $Z_{r-1}^{*}$, both $c\left(Z_{r} \backslash b_{r} ; 2\right)$ and $c\left(Z_{r} \backslash c_{r} ; 2\right)$ are bounded below by 2 . Now consider the representations for $Z_{r} \backslash b_{r}$ and $Z_{r} \backslash c_{r}$ obtained from the matrix $A_{r}$ by deleting the second last and last columns, respectively. Let $H_{1}, H_{2}$ and $H_{3}$ be the hyperplanes of $P G(r-1,2)$ defined by the equations $x_{1}+x_{2}$ $+\cdots+x_{r-1}=0, x_{1}+x_{2}+x_{r}=0$ and $x_{1}+x_{r}=0$. Then $H_{1} \cap H_{2} \cap E\left(Z_{r} \backslash b_{r}\right)$ $=\varnothing$ and $H_{1} \cap H_{3} \cap E\left(Z_{r} \backslash c_{r}\right)=\varnothing$. Thus both $c\left(Z_{r} \backslash b_{r} ; 2\right)$ and $c\left(Z_{r} \backslash c_{r} ; 2\right)$ are bounded above by 2 , so both quantities equal 2 . Moreover, $c\left(Z_{r-1}^{*} ; 2\right)=2$ and $c\left(Z_{r} ; 2\right) \leqslant 3$. The next result completes the proof of the theorem.

(4.3) LEMMA. If $r$ is odd and exceeds 2 , then $c\left(Z_{r} ; 2\right)>2$.

Proof. Consider the matrix $A_{r}$ representing $Z_{r}$. We shall show that $P G(r-1,2)$ does not have two hyperplanes $H$ and $H^{\prime}$ such that $H \cap H^{\prime} \cap E\left(Z_{r}\right)=\varnothing$. Assume that two such hyperplanes do exist. Each hyperplane is defined by an equation of the form $\alpha_{1} x_{1}+\alpha_{1} x_{2}+\cdots+\alpha_{r} x_{r}=0$ where each of $\alpha_{1}, \alpha_{2}, \ldots, \alpha_{r}$ is 0 
or 1 with at least one $\alpha_{i}$ being nonzero. Now we may assume that $H$ avoids $c_{r}$. Then the equation defining $H$ has an odd number of nonzero coefficients, so we may suppose, without loss of generality, that this equation is $x_{1}+x_{2}+\cdots x_{2 k+1}=0$ for some $k \geqslant 0$. The elements of $E\left(Z_{r}\right)$ which are contained in $H$ include $b_{1}, b_{2}, \ldots, b_{2 k+1}, a_{2 k+2}, a_{2 k+3}, \ldots, a_{r}$ and these elements form an odd circuit in $Z_{r}$. Therefore, by Lemma 4.1, $H^{\prime}$ cannot avoid all these elements and we have a contradiction.

The last theorem can be used to show that every loopless binary matroid having no $M\left(\mathscr{W}_{4}\right)$-minor has critical exponent at most 3 . The proof here follows the same lines as the proof of the corresponding result for loopless ternary matroids with no $M\left(\mathscr{W}_{3}\right)$-minor [13, Corollary 3.3]. We omit it since we can obtain a stronger result by combining Theorem 3.3 with the following theorem of Kung.

(4.4) TheOREM [9, Lemma 3.1]. Let $\mathscr{M}$ be a class of simple matroids representable over $G F(q)$ that is closed under restriction. Assume that there is an integer $k$ so that for all $r \geqslant 1$, every rank-r member of $\mathscr{M}$ has at most $k r$ elements. Then, for every member $M$ of $\mathscr{M}, c(M ; q) \leqslant k$.

(4.5) COROllaRY. Let $M$ be a loopless binary matroid having no minor isomorphic to any of $M\left(K_{5}\right), P_{9}$ or $P_{9}^{*}$. Then $c(M ; 2) \leqslant 3$.

Since both $\operatorname{EX}\left(M\left(K_{5}\right), F_{7}\right)$ and $\operatorname{EX}\left(M\left(K_{5}\right), F_{7}{ }^{*}\right)$ are contained in $\operatorname{EX}\left(M\left(K_{5}\right), P_{9}, P_{9}^{*}\right)$, a consequence of the last corollary is the following result of Walton and Welsh [20, Theorem 2, (a) and (b)].

(4.6) Corollary. Let $M$ be a loopless binary matroid. If $M$ is in $\operatorname{EX}\left(M\left(K_{5}\right), F_{7}\right)$ or $\operatorname{EX}\left(M\left(K_{5}\right), F_{7}^{*}\right)$, then $c(M ; 2) \leqslant 3$.

Corollary 4.5 provides a partial answer to Walton and Welsh's question [20, p. 5] as to whether $c(M ; 2) \leqslant 3$ for all loopless binary matroids having no $M\left(K_{5}\right)$-minor. Kung [10] has given another partial answer to this question by showing that for all such $M, c(M ; 2) \leqslant 8$.

ACKNOWLEDGMENT. The author thanks the referee for suggesting that his original method of proof of Theorem 2.1 could be extended to prove Theorem 2.2.

\section{REFERENCES}

1. K. Baclawski and N. L. White, Higher order independence in matroids, J. London Math. Soc. (2) 19 (1979), 193-202.

2. R. E. Bixby and W. H. Cunningham, Short cocircuits in binary matroids (submitted).

3. T. H. Brylawski, A combinatorial model for series-parallel networks, Trans. Amer. Math. Soc. 154 (1971), 1-22.

4. __ A decomposition for combinatorial geometries, Trans. Amer. Math. Soc. 171 (1972), $235-282$.

5. T. H. Brylawski and D. Lucas, Uniquely representable combinatorial geometries, Teorie Combinatorie, Proc. 1973 Internat. Colloq. Accad. Naz. Lincei, Roma, 1976, pp. 83-104.

6. H. H. Crapo and G.-C. Rota, On the foundations of combinatorial theory: Combinatorial geometries, preliminary ed., M.I.T. Press, Cambridge, Mass., 1970.

7. G. A. Dirac, In abstrakten Graphen vorhande vollständige 4-Graphen und ihre Unterteilungen, Math. Nachr. 22 (1960), 61-85.

8. I. Heller, On linear systems with integral valued solutions, Pacific J. Math. 7 (1957), 1351-1364. 
9. J. P. S. Kung, Growth rates and critical exponents of classes of binary combinatorial geometries, Trans. Amer. Math. Soc. 293 (1986), 837-859.

10. , Excluding the cycle geometries of the Kuratowski graphs from binary geometries, Proc. London Math. Soc. (to appear).

11. U. S. R. Murty, Extremal matroids with forbidden restrictions and minors, Proc. Seventh Southeastern Conference on Combinatorics, Graph Theory and Computing, Utilitas Mathematica, Winnipeg, 1976, pp. $463-468$.

12. J. G. Oxley, On 3-connected matroids, Canad. J. Math. 33 (1981), 20-27.

13. , A characterization of the ternary matroids with no $M\left(K_{4}\right)$-minor, J. Combin. Theory Ser. B (to appear).

14. W. R. H. Richardson, Decomposition of chain groups and binary matroids, Proc. Fourth Southeastern Conference on Combinatorics, Graph Theory and Computing, Utilitas Mathematica, Winnipeg, 1973, pp. 463-476.

15. P. D. Seymour, Decomposition of regular matroids, J. Combin. Theory Ser. B 28 (1980), 305-359.

16. Minors of 3-connected matroids, European J. Combin. 6 (1985), 375-382.

17. W. T. Tutte, A homotopy theorem for matroids, I, II, Trans. Amer. Math. Soc. 88 (1958), 144-174.

18. __ Connectivity in matroids, Canad. J. Math. 18 (1966), 1301-1324.

19. P. N. Walton, Some topics in combinatorial theory, D. Phil. thesis, Oxford, 1981.

20. P. N. Walton and D. J. A. Welsh, On the chromatic number of binary matroids, Mathematika 27 (1980), 1-9.

21. D. J. A. Welsh, Matroid theory, Academic Press, London, 1976.

Department of Mathematics, Louisiana State University, Baton Rouge, Louisiana 70803 